in order to noninvasively predict the performance of the device prior to clinical trials. The shunts are characterized mathematically, and simulations are run using estimated parameters for the hydrocephalic patient. Results suggest the relative efficacy of shunt designs in alleviating hydrocephalus and in preventing siphoning or other perturbations of the complex shunt-CSF system. In particular, deterioration of shunt performance due to catheter clogging, material malfunction, and changes in the patient's ability to transport CSF can be modeled. This modeling approach may reduce the number of animal and clinical trials necessary for the testing of devices and can be valuable in rejecting faulty designs and/or refining designs prior to costly mockups and bench testing.

\author{
Eileen Ahearn \\ Biomedical Engineering and \\ Mathematics Curriculum \\ University of North Carolina \\ School of Medicine \\ Chapel Hill, North Carolina \\ June 1988 \\ Received July 8, 1988
}

\title{
COMPUTER ANALYSIS OF THE ELECTROCARDIOGRAM FOR DETECTION OF MYOCARDIAL ISCHEMIA DURING TRANSESOPHAGEAL ATRIAL PACING STRESS
}

Dynamic exercise stress testing remains the major noninvasive diagnostic and prognostic technique in evaluation and management of patients with coronary artery disease. However, not all patients are able to exercise and alternate methods of cardiac stress must be employed. Transesophageal atrial pacing (TAP) has been proposed as an alternative in producing stress in patients who cannot exercise. It offers the advantage of noninvasive direct control over heart rate which is independent of the patient's physical condition. Several computer algorithms have previously been implemented to analyze the electrocardiogram (ECG) during an exercise stress test. However, the presence of large stimulator-induced pacing artifacts confounds computer beat identification, making these methods ineffective during a TAP stress procedure. This research demonstrates the deficiency of present exercise ECG computer methods in processing the ECG during TAP stress and presents novel hardware and software methods which detect and suppress the pacing artifact, detect the $\mathrm{R}$ wave, determine sustained capture, and calculate beat-by-beat and average ST segment level, ST slope, $R$ wave amplitude, instantaneous heart rate, $R R$ and pace- $R$ intervals, and sinus node recovery time upon loss of sustained capture. Five computer methods (single point, average, weighted average, linear least squares, parabolic least squares) were employed in ST segment measurements. A new esophageal electrode as well as new ana$\log$ and digital methodologies including a stimulus artifact suppressor were developed for acquiring the esophageal ventricular ECG during TAP stress. A new vectorcardiographic cardiac-torso model was developed to elucidate theoretical principles underlying observed esophageal ventricular ECG recordings. Results of computer processing of surface and esophageal ventricular ECGs from 6 normal subjects and 
4 patients with known myocardial ischemia during TAP stress are presented. During clinical studies it was observed that TAP duplicates exercise in its ability to produce cardiac stress. It was concluded that both linear and parabolic least squares methods offer simple and accurate computerized ST measurement. The model demonstrated that the esophageal lead is sensitive to both posterior and posteroinferior abnormalities but may not distinguish between them. It was observed that TAP stress causes diagnostic ST segment deviation in the esophageal ventricular ECG in patients with known posteroinferior ischemia.

Hossein Jadvar

College of Engineering University of Michigan Ann Arbor, Michigan June 1988

Received July 26, 1988 